\title{
MicroRNA-181 targets Yin Yang 1 expression and inhibits cervical cancer progression
}

\author{
WEN-YU ZHOU ${ }^{1,2^{*}}$, JIN-CHAN CHEN $^{1 *}$, TING-TING JIAO ${ }^{1}$, NING HUI ${ }^{1}$ and XUAN QI ${ }^{3}$ \\ ${ }^{1}$ Department of Obstetrics and Gynecology, Changhai Hospital, The Second Military Medical University, Shanghai 200433; \\ ${ }^{2}$ Department of Obstetrics and Gynecology, Jiangsu Chinese Armed Police Forces Hospital, Yangzhou, Jiangsu 225000; \\ ${ }^{3}$ Department of Obstetrics and Gynecology, Shanghai Chinese Armed Police Forces Hospital, Shanghai 200051, P.R. China
}

Received November 25, 2013; Accepted June 17, 2014

DOI: $10.3892 / \mathrm{mmr} .2015 .3324$

\begin{abstract}
Dysregulated expression of microRNAs (miRNAs) has been observed in numerous types of human cancer, including cervical cancer (CC). The present study aimed to elucidate the expression and roles of miR-181 in cervical cancer tissues and cells. HeLa cells with a stable overexpression of miR-181 were generated and injected subcutaneously into the front legs of nude mice. Functional assays revealed a reduced rate of proliferation and an enhanced rate of apoptosis following transfection of CC cells with miR-181 mimics. In addition, miR-181 also suppressed tumor growth in the nude mice. At the molecular level, it was found that Yin Yang 1, an oncogene in several types of human cancer, was negatively regulated by miR-181. Therefore, the findings of the present study suggest that exogenous overexpression of miR-181 may be a potential approach for the treatment of $\mathrm{CC}$ in the future.
\end{abstract}

\section{Introduction}

Cervical cancer (CC) has become the second most common female cancer, particularly in developing countries (1). In mainland China, it remains an important public health problem $(2,3)$. Therefore, identification of novel mechanisms of CC may aid the development of strategies for the diagnosis, treatment and determination of prognosis.

Correspondence to: Dr Ning Hui, Department of Obstetrics and Gynecology, Changhai Hospital, The Second Military Medical University, 168 Changhai Road, Shanghai 200433, P.R. China E-mail: 15026810306@163.com

Dr Xuan Qi, Department of Obstetrics and Gynecology, Shanghai Chinese Armed Police Forces Hospital, 4001 Changning Road, Shanghai 200051, P.R. China

E-mail: qixxuan226@sina.com

*Contributed equally

Key words: cervical cancer, cell proliferation, apoptosis, microRNA, miR-181, Yin Yang 1
A previous study has shown that oncogenes and tumor suppressors exhibit critical roles in the development of CC (4). Recent studies also indicate that expression of these genes is tightly regulated by microRNAs (miRNAs), a class of small endogenous RNA molecules $(5,6)$. For example, FoxO1, a tumor suppressor in several types of human cancer, is controlled by miR-96, miR-223 and miR-370 (7-9). Whilst the reason why these distinct miRNAs regulate the same target gene remains unknown, dysregulation of certain miRNAs may contribute to tumor initiation and/or progression. The present study analyzed the expression levels of miR-181 in cervical cancer tissues. Furthermore, the roles and mechanisms of miR-181 in the development of cervical cancer were determined.

\section{Materials and methods}

Tissue samples and cell culture. In total, 30 pairs of samples from CC tissues and the adjacent normal tissues were obtained from patients who underwent surgery in the Department of Obstetrics and Gynecology. This study was approved by the Institutional Review Board of Changhai Hospital, The Second Military Medical University (Shanghai, China). Written informed consent was obtained from each patient. The CC cell lines (HeLa, HeLa-229, SiHa and C33A) and normal cervical epithelial cells (End1/E6E7) were obtained from the Chinese Academy of Sciences Cell Bank (Shanghai, China). Cells were maintained in Dulbecco's modified Eagle's medium (Invitrogen, Carlsbad, CA, USA) supplemented with $10 \%$ fetal bovine serum (Invitrogen). TNF- $\alpha$ (10 ng/ml), IL-1 $\beta$ $(20 \mathrm{ng} / \mathrm{ml})$ and IL-6 $(20 \mathrm{ng} / \mathrm{ml})$ were obtained from Bioyare Company (Shanghai, China).

Quantitative PCR. miRNA Isolation kits (Ambion, Austin, TX, USA) were used to harvest RNA from tissues or cells. Expression of miR-181 was determined by Taqman ${ }^{\circledR}$ MicroRNA assay (Applied Biosystems, Foster City, CA). Quantitative PCR was performed using a 7300 Real-Time PCR system (Applied Biosystems). The PCR conditions included an initial incubation period at $94^{\circ} \mathrm{C}$ for $5 \mathrm{~min}$, followed by a two-step PCR program consisting of $94^{\circ} \mathrm{C}$ for $5 \mathrm{sec}$ and $60^{\circ} \mathrm{C}$ for $30 \mathrm{sec}$, for 40 cycles. The primer sequences for miR-181 were: Forward 5'-AACATTCAACGCTGTCGGTGAAGT-3', and reverse 5'-ACTTCACCGACAGCGTTGAATGTT-3'. All 
of the samples were normalized to the internal control (U6 small nuclear RNA).

Cell proliferation assay. Cell counts were estimated by trypsinizing the cells and performing analysis with a Coulter counter (Beckman Coulter, Fullerton, CA, USA). For BrdU incorporation assays, a cell proliferation enzyme-linked immunosorbent assay (Beyotime, Shanghai, China) was used to analyze the incorporation of BrdU during DNA synthesis following the manufacturer's instructions. Absorbance was measured at $450 \mathrm{~nm}$ in the SpectraMax 190 ELISA reader (Molecular Devices, Sunnyvale, CA, USA).

Cell cycle analysis. The cells were labeled for $15 \mathrm{~min}$ with propidium iodide and immediately analyzed by flow cytometry (Becton Dickinson, San Jose, CA, USA). A total of $\sim 10,000$ cells were acquired and analyzed for DNA content. All of the data were collected, stored and analyzed by ModFit software (http://www.vsh.com/products/mflt/). Histograms represent the percentage of cells in each phase of the cell cycle (G0/G1, S and G2/M).

MicroRNA mimics and transfection. Human miR-181 mimics were purchased from Ambion. The negative controls consist of a scrambled sequence (Ambion). All transfection was performed using Lipofectamine 2000 (Invitrogen) following the manufacturer's instructions.

Western blot analysis. Cells were harvested and lysed with ice-cold lysis buffer $(50 \mathrm{~mm}$ Tris- $\mathrm{HCl}, \mathrm{pH} 6.8,181 \mathrm{~mm}$ 2-mercaptoethanol, $2 \%$ w/v SDS, $10 \%$ glycerol; Biosune Biotechnology Co., Ltd., Shanghai, China). Following centrifugation at $10,000 \times \mathrm{x}$ and $4^{\circ} \mathrm{C}$ for $15 \mathrm{~min}$, proteins in the supernatants were quantified and separated by $10 \%$ SDS-PAGE and transferred to a nitrocellulose membrane (Amersham Bioscience, Amersham, UK). After blocking with $10 \%$ nonfat milk in phosphate-buffered saline, membranes were immunoblotted with antibodies as indicated, followed by horseradish peroxidase-linked anti-mouse or anti-rabbit IgG secondary antibodies (Cell Signaling Technology, Inc., Beverly, MA, USA). The signals were detected by SuperSignal West Pico Chemiluminescent Substrate kit (Pierce, Rockford, IL, USA) according to the manufacturer's instructions. Polyclonal rabbit anti-YY1, polyclonal rabbit p53 and polyclonal mouse C-myc antibodies were purchased from Abcam (Cambridge, MA, USA). Protein levels were normalized to GAPDH (polyclonal mouse; Santa Cruz Biotechnology, Inc., Santa Cruz, CA, USA).

Luciferase reporter assay. The wild-type and mutant 3'-UTR fragments of the YY1 gene were cloned into pMir-Report (Ambion). Mutations were introduced in potential miR-181 binding sites using the QuikChange Site-Directed Mutagenesis kit (Stratagene, La Jolla, CA, USA). Luciferase values were determined using the Dual-Luciferase Reporter Assay system (Promega, Madison, WI, USA).

Tumor growth assay. Male BALB/c nude mice aged 4 weeks old were purchased from Shanghai Laboratory Animal Company (Shanghai, China). HeLa cells $\left(2 \times 10^{5}\right)$, either
A

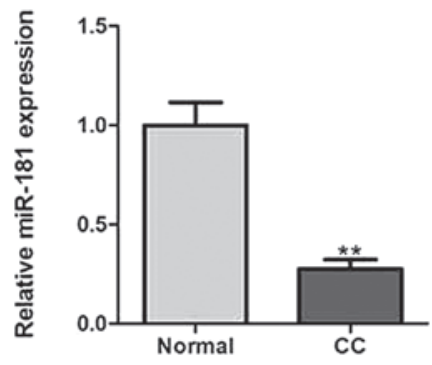

B

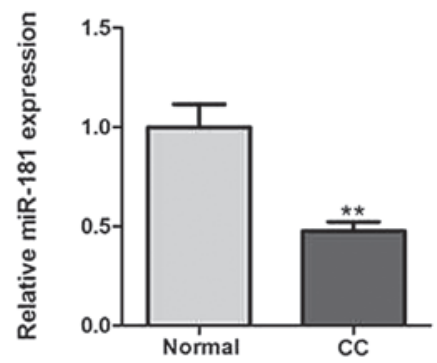

Figure 1. Expression levels of miR-181 in cervical cancer (CC) tissues. (A) miR-181 expression levels were determined by quantitative PCR in human $\mathrm{CC}$ tissues and adjacent noncancerous tissues. (B) Circulating miR-181 expression levels in patients with CC and controls. ${ }^{* *} \mathrm{P}<0.01 \mathrm{com}-$ pared with normal tissues. $\mathrm{N}=30$.

stably expressing miR-181 or negative controls, were injected subcutaneously into the front legs of the mouse. The mice were observed over 5 weeks for tumor formation. The mice were then sacrificed by cervical dislocation, the tumors were recovered and the wet weight of each tumor was determined.

Statistical analysis. Differences between groups were analyzed using Student's t-test analysis and expressed as the mean \pm standard error from at least three separate experiments. $\mathrm{P}<0.05$ was considered to indicate a statistically significant difference.

\section{Results}

miR-181 is downregulated in CC tissues and cells. Expression of miR-181 in CC tissues was compared to that in the adjacent normal tissues using quantitative PCR. As a result, it was revealed that miR-181 expression levels were greatly reduced in CC tissues (Fig. 1A). The circulating levels of miR-181 were quantified in the serum of CC patients and healthy controls, and a significant difference was also observed between the two groups (Fig. 1B).

Inflammatory cytokines inhibit miR-181 expression. To investigate the reasons for the downregulation of miR-181, its expression was determined in CC cell lines and normal cervical cells. The expression levels of miR-181 in four CC cell lines (HeLa, HeLa-229, SiHa and C33A), was observed to be lower compared with those in the normal cervical cell line (End1/E6E7) (Fig. 2A).

Previous studies have demonstrated that inflammatory responses are responsible for the high rate of $\mathrm{CC}$ development $(10,11)$. Therefore, End1/E6E7 cells were treated with several pro-inflammatory cytokines. In the present study TNF $\alpha$, IL- $1 \beta$ and IL- 6 significantly repressed miR-181 expression in a dose-dependent manner (Fig. 2B). Furthermore, 
A

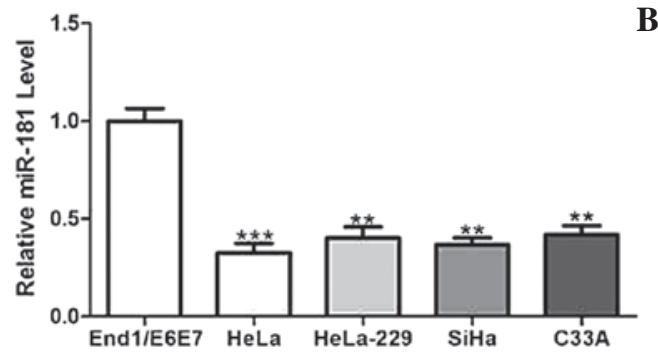

C

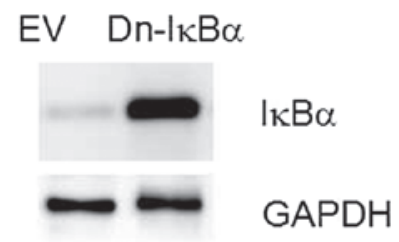

B

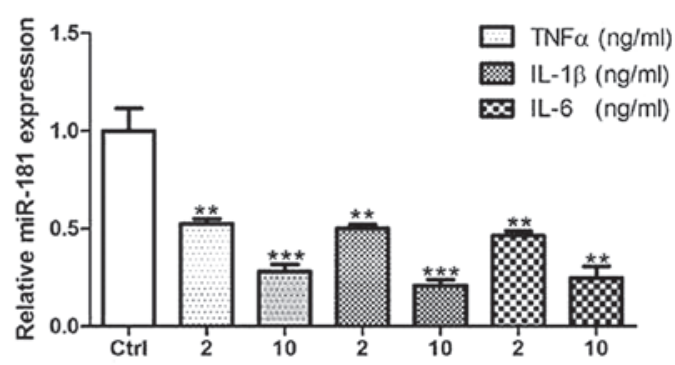

D

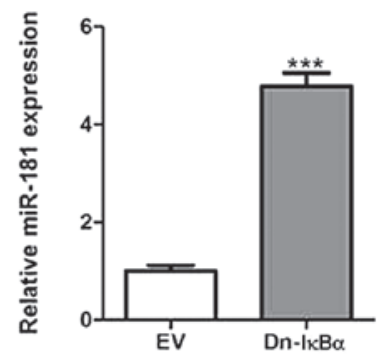

Figure 2. Inflammatory cytokines inhibit miR-181 expression. (A) miR-181 expression levels in normal cervical epithelial cells (End1/E6E7) and cervical cancer cell lines (HeLa, HeLa-229, SiHa and C33A); (B) miR-181 expression levels in End1/E6E7 cells treated with TNF $\alpha$, IL-1 $\beta$, IL-6 or vehicle control (Ctrl)

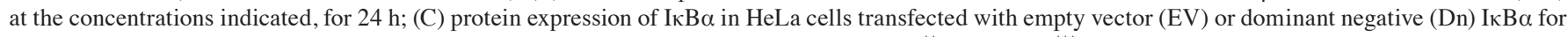
$24 \mathrm{~h}$; (D) miR-181 expression levels in HeLa cells transfected with EV or Dn-IкB $\alpha$ for 36 h. ${ }^{* * *} \mathrm{P}<0.01$ and ${ }^{* * * *} \mathrm{P}<0.001$ compared with control cells.

A

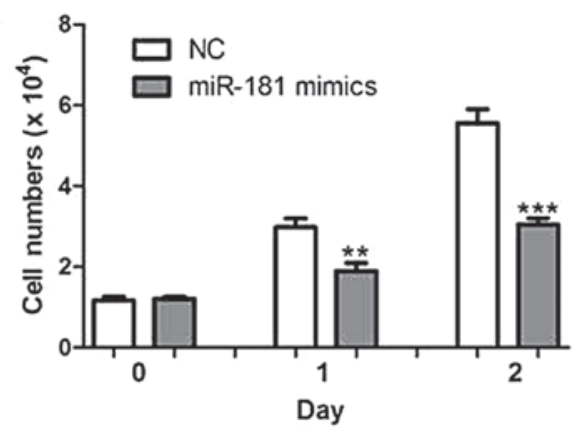

C

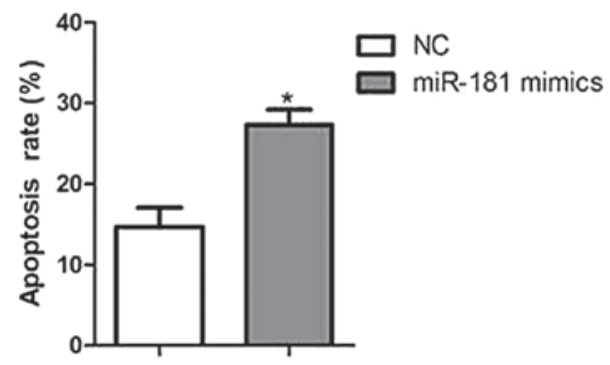

$\mathbf{B}$

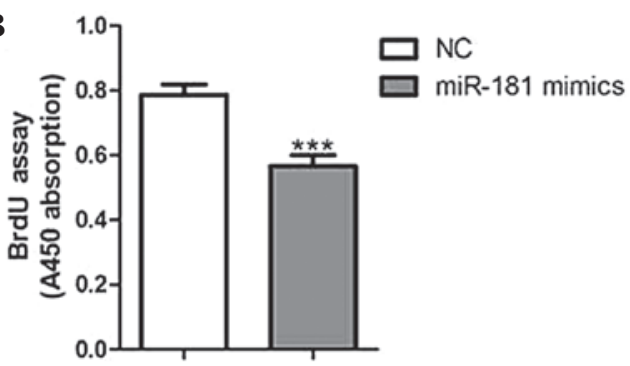

D

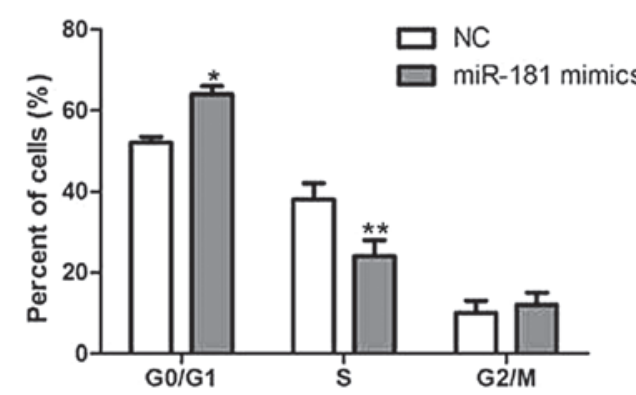

Figure 3. miR-181 overexpression inhibits cervical cancer cell proliferation. (A) The growth of HeLa cells after transfection of miR-181 mimics or negative control (NC); (B and C) the proliferative potential (BrdU) assays and apoptotic rate were determined in HeLa cells transfected with miR-181 mimics or NC; (D) the cell cycle phase of HeLa cells transfected with miR-181 mimics or NC were analyzed by flow cytometry. $\mathrm{P}<0.05,{ }^{* * *} \mathrm{P}<0.01$ and ${ }^{* * * *} \mathrm{P}<0.001$ compared with control cells.

HeLa cells were transfected with plasmids expressing a dominant negative (Dn) I $\mathrm{KB} \alpha$ (Fig. 2C), which could bind with NF- $\kappa \mathrm{B}$ and inhibit NF- $\kappa \mathrm{B}-$ mediated inflammatory signaling (12). As expected, Dn-IкB $\alpha$ significantly upregulated miR-181 expression levels in HeLa cells compared with those in the controls (Fig. 2D). These results suggest that the downregulation of miR-181 in CC tissues and cell lines may be due to, at least in part, the persistent activation of inflammatory response.
miR-181 overexpression inhibits CC cell proliferation in vitro. To determine the effect of miR-181 on CC cell growth, HeLa cells were transfected with either miR-181 mimics or negative controls. As a result, cell growth was significantly reduced in miR-181-overexpressed cells compared with that of their corresponding controls (Fig. 3A). Furthermore, cells overexpressing miR-181 had a lower rate of proliferation and a higher rate of apoptosis (Fig. 3B and C). Cell cycle analysis of miR-181-overexpressed cells showed a significant increase in the percentage 

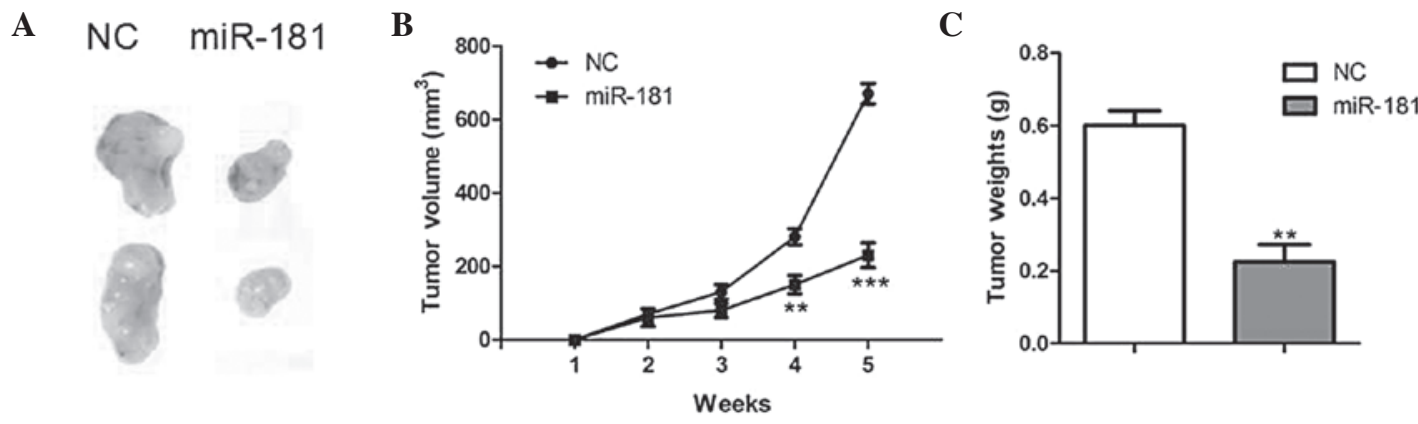

Figure 4. miR-181 overexpression inhibits tumor growth in vivo. HeLa cells stably transfected with miR-181 or negative control (NC) were injected into nude mice ( $n=5$ for each group) and followed up for tumorigenesis. (A) Representative images, (B) growth curve of tumor volumes, (C) tumor weights five weeks after injection.

A

$$
\begin{array}{ll}
\text { YY13'UTR(Wild-type) } & \text { 5'-UCGAUACUGGACUACUACACUUCA-3' } \\
\text { miR-181 } & \text { 3'-AACAUUCAACGCUGUCGGUGAAGU-5' } \\
\text { YY13'UTR(Mutant) } & \text { 5'-UCGAUACUGGACUACUACACGGCA-3' }
\end{array}
$$

B

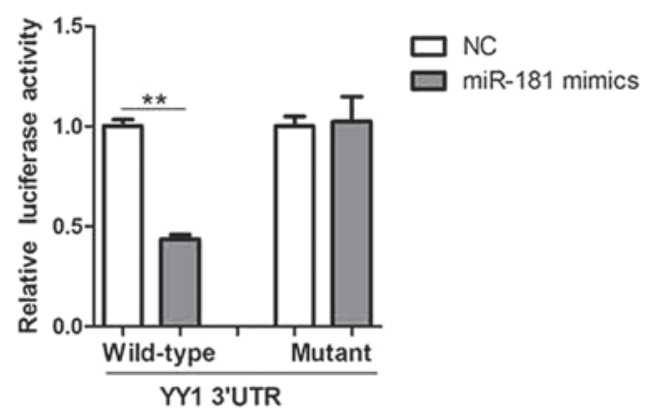

C NC miR-181 mimics

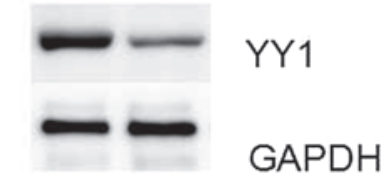

Figure 5. miR-181 represses Yin Yang 1 (YY1) expression in HeLa cells. (A) Prediction of miR-181 binding sites in the 3'-UTRs of human YY1 gene by TargetScan software. Potential binding site is highlighted in bold; (B) luciferase reporter assays in HeLa cells. Cells were transfected with wild-type or mutant 3'-UTR-reporter constructs together with miR-181 mimics or negative control (NC); (C) protein levels of YY1 were determined by western blot analysis in HeLa cells transfected with miR-181 mimics or NC.

A

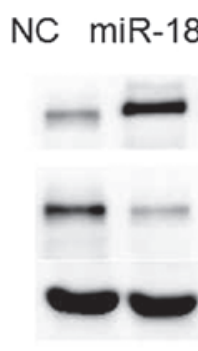

B

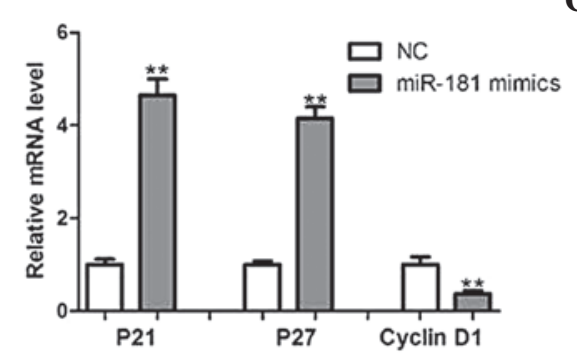

C NC $\operatorname{miR}-181$

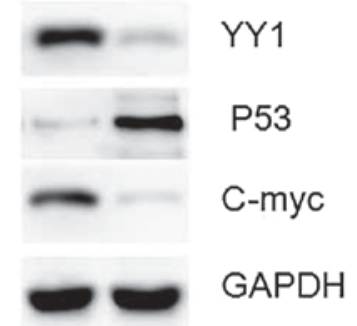

Figure 6. miR-181 regulates downstream target genes of Yin Yang 1 (YY1). (A) Protein levels of P53 and C-myc were determined by western blot analysis in HeLa cells transfected with miR-181 mimics or negative control (NC); (B) mRNA levels of P21, P27 and Cyclin D1 were analyzed by quantitative PCR in HeLa cells transfected with miR-181 mimics or NC; (C) protein levels of YY1, P53 and C-myc were determined by western blot analysis in tumors stably transfected with miR-181 or NC.

of cells in G0/G1 phase and a reduction of those in S phase (Fig. 3D).

miR-181 overexpression inhibits tumor growth in vivo. To further determine the roles of miR-181, HeLa cells with stable overexpression of miR-181 were generated and injected subcutaneously into the front legs of the nude mice. The tumor growth was closely monitored for 5 weeks. After this time the tumor size and weight were markedly reduced in miR-181-overexpressed tumors in comparison with the control tumors (Fig. 4A and B), suggesting that miR-181 suppresses $\mathrm{CC}$ growth in vivo. 
miR-181 targets YY1 3'-UTR and downregulates its expression. To understand the underlying mechanism of growth inhibition by miR-181, potential targets of miR-181 were searched using TargetScan (www.targetscan.org) and miRWalk (www.umm.uni-heidelberg.de/apps/zmf/mirwalk/) software. It was identified that YY1, an oncogene in human cancer, harbored a potential miR-181 binding site (Fig. 5A). To verify whether YY1 is a direct target of miR-181, luciferase report vectors that contained the putative miR-181 binding sites within YY1 3'-UTR were constructed. The overexpression of miR-181 significantly repressed the luciferase activity when the reporter construct contained the YY1 3'-UTR in HeLa cells (Fig. 5B). However, mutation of the miR-181 binding site from the YY1 3'-UTR eliminated this effect of miR-181, suggesting that miR-181 directly inhibited YY1 expression by targeting its 3'-UTR (Fig. 5B). The miR-181 precursor inhibited the protein expression of YY1 as indicated by western blot analysis (Fig. 5C), whilst its mRNA levels remained unchanged (data not shown). Therefore, these results suggest that miR-181 negatively regulates YY1 expression at the translational level.

YY1 has been reported to downregulate p53 expression whilst upregulating $\mathrm{C}$-myc,which are key regulators of tumor growth and cell cycle progression $(13,14)$. In HeLa cells overexpressing miR-181 in the present study, an increase in the expression levels of p53 was observed, as well as the downregulation of C-myc, as compared with levels in controls (Fig. 6A). Additionally, mRNA levels of P21 and P27, downstream targets of P53, were increased, while Cyclin D1, a target of C-myc, was repressed (Fig. 6B). The levels of YY1, p53 and C-myc were also affected in tumors overexpressing miR-181, when compared with the levels in negative controls (Fig. 6C). These results confirm that YY1 is an important target gene of miR-181 in CC.

\section{Discussion}

It has been demonstrated that several miRNAs are dysregulated in CC tissues or cell lines, including miR-20, miR-196 and miR-203 (15-17). In the present study, through experiments in vitro and in vivo, the role of miR-181 in CC was determined. It was found that miR-181 overexpression was able to inhibit cell proliferation and induce apoptosis and cell cycle arrest in G1 phase. Furthermore, miR-181 was able to inhibit tumor growth in the nude mice. Therefore, miR-181 may be a tumor suppressor in the development of CC; however further studies with larger clinical samples are required.

Previous studies have shown that miR-181 also functions as a tumor suppressor which triggers growth inhibition, induces apoptosis and inhibits invasion in glioma cells (18). However, transforming growth factor TGF- $\beta$-mediated upregulation of hepatic miR-181 promoted growth, clonogenic survival, migration and invasion of hepatocellular carcinoma cells (19). Although the inconsistency in the roles of miR-181 remains unexplained, the specificity of the roles of miR-181 may depend on its target genes and/or cellular context.

YY1 was further experimentally validated as a miR-181 target in CC in the present study. YY1, a transcription factor of the polycomb group protein family, is widely expressed in various tissues and participates in development, metabolism and tumorigenesis (20-22). A study into YY1 expression in tumor tissues revealed that YY1 overexpression may have an important role in the regulation of sensitivity and resistance of tumor cells to chemotherapy and immunotherapy (23). At the molecular level, a previous study supports the role of YY1 in tumorigenesis via its association with the tumor suppressor gene P53 (13). YY1 may interact with P53 and promote its protein degradation by blocking p300-dependent p53 acetylation and stabilization (13). YY1 may also activate C-myc and Cyclin D1 promoters to enhance their transcription (23). Therefore, inhibitors, antisense therapies, and silencer RNAs that target YY1 may serve as potential therapies for cancer progression.

In conclusion, the key finding of the present study highlights the theory that overexpression of miR-181 inhibits CC cell growth in vitro and in vivo by regulating YY1 expression. Therefore, the results regarding the functional interaction of miR-181 and YY1 signaling in CC may aid the development of novel gene therapies in the future.

\section{References}

1. Meijer CJ and Berkhof J: Screening: Cervical cancer - should we abandon cytology for screening? Nat Rev Clin Oncol 9: 558-559, 2012.

2. Li J, Kang LN and Qiao YL: Review of the cervical cancer disease burden in mainland China. Asian Pac J Cancer Prev 12: 1149-1153, 2011.

3. Shi JF, Canfell K, Lew JB and Qiao YL: The burden of cervical cancer in China: synthesis of the evidence. Int J Cancer 130: 641-652, 2012.

4. Hung CF, Wu TC, Monie A and Roden R: Antigen-specific immunotherapy of cervical and ovarian cancer. Immunol Rev 222: 43-69, 2008.

5. Ameres SL and Zamore PD: Diversifying microRNA sequence and function. Nat Rev Mol Cell Biol 14: 475-488, 2013.

6. Sun K and Lai EC: Adult-specific functions of animal microRNAs. Nat Rev Genet 14: 535-548, 2013.

7. Haflidadóttir BS, Larne O, Martin M, et al: Upregulation of miR-96 enhances cellular proliferation of prostate cancer cells through FOXO1. PLoS One 8: e72400, 2013.

8. Wu L, Li H, Jia CY, et al: MicroRNA-223 regulates FOXO1 expression and cell proliferation. FEBS Lett 586: 1038-1043, 2012.

9. Wu Z, Sun H, Zeng W, He J and Mao X: Upregulation of MircoRNA-370 induces proliferation in human prostate cancer cells by downregulating the transcription factor FOXO1. PLoS One 7: e45825, 2012.

10. Mai CW, Kang YB and Pichika MR: Should a Toll-like receptor 4 (TLR-4) agonist or antagonist be designed to treat cancer? TLR-4: its expression and effects in the ten most common cancers. Onco Targets Ther 6:1573-1587, 2013.

11. Deivendran S, Marzook KH and Radhakrishna Pillai M: The role of inflammation in cervical cancer. Adv Exp Med Biol 816: 377-399, 2014.

12. Ding G, Liu HD, Huang Q, et al: HDAC6 promotes hepatocellular carcinoma progression by inhibiting P53 transcriptional activity. FEBS Lett 587: 880-886, 2013.

13. Grönroos E, Terentiev AA, Punga T and Ericsson J: YY1 inhibits the activation of the p53 tumor suppressor in response to genotoxic stress. Proc Natl Acad Sci USA 101: 12165-12170, 2004.

14. Liao WR, Hsieh RH, Hsu KW, et al: The CBF1-independent Notch1 signal pathway activates human C-myc expression partially via transcription factor YY1. Carcinogenesis 28: 1867-1876, 2007.

15. Zhao S, Yao D, Chen J and Ding N: Circulating miRNA-20a and miRNA-203 for screening lymph node metastasis in early stage cervical cancer. Genet Test Mol Biomarkers 17: 631-636, 2013.

16. How C, Hui AB, Alajez NM, et al: MicroRNA-196b regulates the homeobox B7-vascular endothelial growth factor ax is in cervical cancer. PLoS One 8: e67846, 2013. 
17. Zhu X, Er K, Mao C, et al: miR-203 suppresses tumor growth and angiogenesis by targeting VEGFA in cervical cancer. Cell Physiol Biochem 32: 64-73, 2013.

18. Shi L, Cheng Z, Zhang J, et al: hsa-mir-181a and hsa-mir-181b function as tumor suppressors in human glioma cells. Brain Res 1236: 185-193, 2008.

19. Wang B, Hsu SH, Majumder S, et al: TGFbeta-mediated upregulation of hepatic miR-181b promotes hepatocarcinogenesis by targeting TIMP3. Oncogene 29: 1787-1797, 2010.

20. Donohoe ME, Zhang X, McGinnis L, et al: Targeted disruption of mouse Yin Yang 1 transcription factor results in peri-implantation lethality. Mol Cell Biol 19: 7237-7244, 1999.
21. Lu Y, Xiong X, Wang X, et al: Yin Yang 1 promotes hepatic gluconeogenesis through upregulation of glucocorticoid receptor. Diabetes 62: 1064-1073, 2013.

22. Wang H, Garzon R, Sun H, et al: NF-kappaB-YY1-miR-29 regulatory circuitry in skeletal myogenesis and rhabdomyosarcoma. Cancer Cell 14: 369-381, 2008.

23. Gordon S, Akopyan G, Garban H and Bonavida B: Transcription factor YY1: structure, function, and therapeutic implications in cancer biology. Oncogene 25: 1125-1142, 2006. 